\title{
SISTEMATIZAÇÃO DA ASSISTÊNCIA DE ENFERMAGEM COMO FERRAMENTA DA GERÊNCIA DO CUIDADO: ESTUDO DE CASO
}

\author{
Systematization of nursing assistance as a care management tool: case study \\ Sistematización de la asistencia de enfermería como herramienta de la gerencia del \\ cuidado: estudio de caso.
}

Érica Torres ${ }^{1}$
Zenith Rosa Silvino $^{4}$

\author{
Bárbara Pompeu Christovam² \\ Marilda Andrade ${ }^{5}$
}

Patrícia Claro dos Santos Fuly ${ }^{3}$

\section{RESUMO}

Trata-se de estudo de natureza qualitativa, do tipo estudo de caso único, em que se adotou uma triangulação de técnicas para obtenção dos dados (questionário estruturado, observação sistemática e análise documental) e análise de conteúdo segundo Bardin para análise. Objetivou-se verificar se os enfermeiros do Serviço de Infectologia de um hospital universitário consideram a Sistematização da Assistência de Enfermagem (SAE) uma ferramenta da gerência do cuidado e analisar se a SAE, enquanto ferramenta gerencial, serve de base para suas ações de cuidar. Identificou-se que os sujeitos compreendem o que é SAE e a gerência do cuidado e enxergam uma relação entre as duas. Ficou demonstrada uma lacuna entre o que é dito e o que é praticado. Não se pode considerar que a SAE, enquanto ferramenta gerencial, serve de base para o cuidado. Recomendam-se estratégias que possibilitariam um feedback às gerências dos serviços de enfermagem sobre estes aspectos.

Palavras-chave: Pesquisa em Administração de Enfermagem. Processos de enfermagem. Doenças transmissíveis.

\begin{abstract}
It's about a qualitative study, a single case study type, where was adopted a triangulation of techniques in order to obtain data collection (structured questionnaire, systematic observation and documental analysis) and content analysis according to Bardin for analysis. The objective was to determine whether the nurses of the infectious diseases of a university hospital consider the Systematization of nursing assistance (SNA) as a tool for care management and examine whether the SNA as a management instrument, provides the basis for their actions of caring. It was identified that the subjects understand what the SNA is and what the care management means, and that they see a relationship between both of them. It was detected that there is a gap between what is said and what is really practiced. It's not possible to consider that the SNA, as a management tool, provides the basis for caring. Strategies that allow the management of nursing services to get a feedback on these aspects are recommended.
\end{abstract}

Keywords: Nursing Administration Research. Nursing Process. Communicable Diseases.

\section{Resumen}

Se trata de un estudio de naturaleza cualitativa, de caso único, en el cual se adoptó una triangulación de técnicas para la recolección de datos (cuestionario estructurado, observación sistemática y análisis de documentos) y análisis de contenido según Bardin. El objetivo fue determinar si el personal de enfermería del sector de enfermedades infecciosas de un hospital universitario considera la Sistematización de la Asistencia de Enfermería (SAE) como herramienta para la gerencia del cuidado y examinar si la SAE, como instrumento de gestión, proporciona la base para sus acciones de cuidado. Se ha identificado que los sujetos comprenden qué es SAE y qué es gerencia del cuidado y que entienden una relación entre ambos. Se demostró una diferencia entre lo que se dice y lo que se practica. No se puede considerar que la SAE como instrumento de gestión proporcione la base para su cuidado. Se recomiendan estrategias que permitan a las gerencias obtener un feedback de los servicios de enfermería en estos aspectos.

Palabras clave: Investigación en Administración de Enfermería. Procesos de Enfermería. Enfermedades Trasmisibles.

\footnotetext{
'Enfermeira. Mestranda em Ciências do Cuidado em Saúde pela UFF. Fiscal do Conselho Regional de Enfermagem do Rio de Janeiro (COREN-RU). Membro do NUPGES - UFF e do GESAE-UFF. Barra Mansa - R. Brasil. E-mail: kinhatorres@bol.com.br. ${ }^{2}$ Enfermeira. Doutora em Enfermagem. Professora Adjunta do departamento de Fundamentos de Enfermagem e Administração da Escola de Enfermagem Aurora de Afonso Costa/UFF. Coordenadora do curso de graduação em enfermagem e licenciatura da Escola de Enfermagem Aurora de Afonso Costa/UFF. Vice-Coordenadora do Núcleo de Estudos e Pesquisas em Cidadania e Gerência na Enfermagem e Vice-Líder do Grupo de Pesquisa Cidadania e Gerência na Enfermagem. Niterói - RJ. Brasil. E-mail: babypompeu@gmail.com; ${ }^{3}$ Enfermeira. Doutora em Enfermagem. Professora Adjunta do departamento de Enfermagem Médico-cirúrgica da Escola de Enfermagem Aurora de Afonso Costa/UFF. Membro do GESAE-UFF. Rio de Janeiro - R. Brasil. E-mail: claropatricia@yahoo.com.br.; ${ }^{4}$ Enfermeira. Doutora em Enfermagem. Professora Titular do departamento de Fundamentos de Enfermagem e Administração da Escola de Enfermagem Aurora de Afonso Costa/UFF. Coordenadora do Mestrado Profissional em Enfermagem Assistencial. Niterói - RJ. Brasil. E-mail: zenithrosa@terra.com.br;'5nfermeira. Doutora em Enfermagem. Professora Associada do departamento de Enfermagem Médico-cirúrgica da Escola de Enfermagem Aurora de Afonso Costa/UFF. Vice-coordenadora geral da pós-graduação da Escola de Enfermagem Aurora de Afonso Costa/UFF. Niterói - R.. Brasil. E-mail: marildaandrade@uol.com.br.
} 


\section{INTRODUÇÃO}

0 enfermeiro gerente é o profissional que assume a coordenação do trabalho da equipe de enfermagem. Seu trabalho envolve ações de cuidado direto e indireto, ou seja, volta-se para a Gerência do Cuidado, possibilitando o desenvolvimento de uma prática profissional diferenciada. É cada dia mais cobrada desses profissionais a habilidade de trabalhar em equipe considerando as necessidades da mesma, bem como de seus clientes, familiares e da própria instituição.

Sendo o exercício profissional da enfermagem assegurado pelo Decreto $94.406 / 87$ que regulamenta a Lei 7.498/86, destaca-se a alínea c, parágrafo I do artigo $8^{\circ}$, que versa sobre as atividades privativas dos enfermeiros, a saber "é tarefa privativa do enfermeiro o planejamento, organização, coordenação, execução e avaliação dos serviços da assistência de enfermagem" $1: 2$

Nota-se a semelhança entre 0 artigo exposto e 0 conceito de gerência do cuidado, que a aponta como uma relação dialética entre o saber-fazer gerenciar e o saber-fazer cuidar. As ações de gerência do cuidado de enfermagem caracterizam-se por ações expressivas e instrumentais de cuidado direto e indireto, a articulação e a interface dos aspectos técnicos, políticos e da politicidade, social, comunicativo, de desenvolvimento da cidadania e organizacionais. ${ }^{2}$

Considera-se que o enfermeiro gerencia o cuidado quando o planeja, quando o delega ou o faz, quando prevê e provê recursos, capacita sua equipe, educa o usuário, interage com outros profissionais e quando ocupa espaços para que consiga concretizar melhorias no cuidado, sendo fundamental que se aproprie dos instrumentos gerenciais para transformar o processo de cuidar. ${ }^{2}$

A Sistematização da Assistência de Enfermagem (SAE), reconhecida pelos profissionais de enfermagem como marco a ser institucionalizado nos serviços de saúde, é uma importante ferramenta gerencial utilizada para planejamento, execução, controle e avaliação das ações de cuidado direto e indireto aos clientes. $^{3,4}$

Originalmente regida pela Resolução do Conselho Federal de Enfermagem (COFEN) 272/2002 e atualmente pela Resolução COFEN 358/2009, a SAE é utilizada como metodologia assistencial entendida como aplicação prática de uma teoria de enfermagem na assistência aos clientes, utilizando ou não o Processo de Enfermagem (PE) ou partes do mesmo. Ela é todo o planejamento registrado da assistência que abrange desde a criação e implementação do manual de normas e rotinas das unidades à descrição padronizada, até a adoção do PE. ${ }^{4}$

A problemática desse estudo surge através do pressuposto de que a gerência de enfermagem não consegue avaliar os resultados de sua equipe quando não há um método sistematizado de assistência, tendo como temática a gerência do cuidado pautada na SAE aos clientes internados no Serviço de Infectologia de um hospital público e universitário no município de Niterói-RJ.

Uma proposta de implantação da SAE vem sendo desenvolvida desde 1995 na instituição estudada, com o caráter de obrigatoriedade. A base da conceituação da SAE nesse hospital conta com o apor te conceitual de Wanda Horta, mas, no entanto, sua operacionalização é baseada na metodologia da assistência de Lygia Paim, que preconiza três fases básicas, a saber: (1) identificação dos problemas no histórico de enfermagem, (2) prescrição de enfermagem e (3) evolução de enfermagem. ${ }^{5} \mathrm{~A}$ própria Paim apoia sua metodologia em Horta, porém "seu processo é mais enxuto, com apenas três etapas básicas". 6:11 É nessa perspectiva que se desenvolve essa pesquisa.

Os objetivos foram: verificar se os enfermeiros do Serviço de Infectologia consideram a SAE como ferramenta da gerência do cuidado e analisar se a SAE, enquanto uma ferramenta da gerência do cuidado, serve de base para as ações de cuidar no Serviço de Infectologia.

\section{METODOLOGIA}

Trata-se de um estudo qualitativo, do tipo estudo de caso único, que é uma investigação sobre uma única situação, em que se busca um aprofundamento dos dados, sem preocupação sobre a frequência de sua ocorrência. Esta metodologia deve ser adotada quando se quer estudar algo singular, que tenha valor em si mesmo. ${ }^{7}$ Essa abordagem se encaixou com os propósitos do estudo. Visando garantir confiabilidade, foi adotada uma triangulação de técnicas para obtenção dos dados, a saber: questionário estruturado, observação sistemática e análise documental. A triangulação em um estudo de caso é um fundamento lógico que corresponde ao uso de diferentes métodos para examinar o mesmo fenômeno sob várias perspectivas.?

Os sujeitos da pesquisa foram todos os seis enfermeiros do Serviço de Infectologia, sendo um diarista, dois plantonistas diurnos e três plantonistas noturnos. Todos assinaram o termo de consentimento livre e esclarecido, participando voluntariamente da pesquisa. Os mesmos foram caracterizados na pesquisa por codinomes de árvores frutíferas a fim de preservar suas identidades. Cabe ressaltar que foram cumpridas todas as especificações da resolução 196/96, do Ministério da Saúde, sendo a pesquisa aprovada pelo Comitê de Ética e Pesquisa do Hospital Universitário Antônio Pedro, da Universidade Federal Fluminense, com o CEP CMM/HUAP n ${ }^{0}$ 259/08 e CAAE 0203.0.258.000-08.

0 questionário estruturado continha três partes e foi aplicado em momento oportuno aos sujeitos da pesquisa, escolhido pelos mesmos de acordo com suas disponibilidades, no início ou término de seus turnos de trabalho. A primeira parte, direcionada para a identificação e caracterização dos sujeitos, continha espaço para registro do nome, codinome (escolhido pelo pesquisador, com 
consentimento do sujeito), escala de trabalho, turno e tempo de trabalho no setor.

A segunda parte continha quatro perguntas abertas, a saber: (1) 0 que entende por Sistematização da Assistência de Enfermagem?, (2) 0 que entende por Gerência do Cuidado?, (3) Vê alguma relação entre as duas? Qual? e (4) Sistematiza a assistência que presta na sua unidade de trabalho, sim ou não? Se sim, utiliza alguma teoria de enfermagem para embasar tais ações? Qual?, relativas à compreensão dos sujeitos acerca da SAE e gerência do cuidado, relação entre os dois temas e utilização da SAE.

A terceira parte do questionário era referente a uma escala de Likert, utilizada para medir a utilização da metodologia da assistência segundo Paim. A escala de Likert tem a finalidade de atribuir um escore numérico aos atributos/elementos que estão sendo medidos em uma sequência..$^{8}$ Os critérios da escala de Likert se referiam ao histórico de enfermagem, prescrições de enfermagem e evolução de enfermagem. Receberam pontuações seguindo a legenda: (5) pontos = realiza muitas vezes; (4) pontos = realiza; (3) pontos $=$ realiza poucas vezes; (2) pontos $=$ realiza com dificuldade; e (1) ponto = não realiza. 0 escore máximo possível foi de quinze e o mínimo de três pontos.

A segunda técnica foi a observação sistemática. Nesse tipo de observação o rigor científico é indispensável. ${ }^{8}$ Elaborou-se um roteiro composto por duas partes. A primeira continha tópicos abertos e fechados que guiaram a observação; a segunda era composta pela mesma escala de Likert construída no questionário. 0 objetivo da repetição da escala foi confrontar, na análise, as respostas oferecidas pelos sujeitos com as obtidas pela observação do pesquisador.

A última técnica foi a análise documental retrospectiva de prontuários. Para tornar a análise efetiva, adotou-se um instrumento denominado Auditoria de Prontuários, um formulário estruturado para investigar vestígios da SAE nos registros de enfermagem. Foram analisados treze prontuários provenientes do Serviço de Infectologia, escolhidos aleatoriamente, respeitando os critérios de inclusão e exclusão, que foram: (1) deveriam ter sido abertos há no máximo um ano, (2) deveriam se encontrar fechados. A quantidade de prontuários analisados foi definida adotando-se o critério de saturação dos dados.

Analisar as evidências de um estudo de caso é uma atividade particularmente difícil, pois as estratégias e as técnicas não têm sido bem definidas. Durante a análise a pesquisa qualitativa pode se valer de técnicas estatísticas que contribuem para verificar informações e reinterpretar observações, sem perder seu foco qualitativo, permitindo conclusões menos objetivas. ${ }^{9} \mathrm{~A}$ análise foi realizada de forma diferenciada, conforme explicitado abaixo.
As informações das quatro perguntas do questionário foram analisadas através da Análise de Conteúdo que "é um conjunto de técnicas de análise das comunicações, que utiliza procedimentos sistemáticos e objetivos de descrição do conteúdo das mensagens". 10:33 Com base nessa técnica, a partir do conteúdo bruto das informações, foi possível identificar os núcleos de sentido presentes nas comunicações dos sujeitos e a frequência (número de vezes) com que os mesmos surgiram, sendo 6 o maior número e 10 menor.

Foram somados manualmente os escores das Escalas de Likert e atribuídos graus de realização das ações. Os itens das escalas foram classificados em: ALTO - para escores superiores ou iguais a 11; INTERMEDIÁRI0 - para escores com valores entre 6 e 11 ; e BAIXO - para escores com valores abaixo ou iguais a 6 .

Por fim, os dados da auditoria de prontuários foram apresentados com estatística descritiva simples e descrição dos achados, confrontados com os achados presentes na literatura revisada e com os dados do questionário. Por fim, os dados da observação sistemática foram, assim como os demais, confrontados com os dados do questionário.

\section{RESULTADOS E DISCUSSÃO}

Diante dos objetivos traçados para a pesquisa e dos dados obtidos na pesquisa que foram confrontados entre si, surgiram duas categorias empíricas, a saber: (1) Sistematização da assistência de enfermagem e sua utilização no Serviço de Infectologia e (2) Gerência do cuidado e sua relação com a Sistematização da assistência de enfermagem.

\section{Sistematização da assistência de enfermagem e sua utilização no Serviço de Infectologia}

Essa categoria refere-se à compreensão dos sujeitos acerca da SAE e sua utilização no Serviço de Infectologia. Abrange o confronto dos dados obtidos através das questões um e quatro do questionário, além da auditoria de prontuários, as escalas de Likert e comparação com a observação sistemática dos sujeitos.

Os núcleos de sentido que emergiram da questão 1 e as respectivas frequências com que surgiram foram: organização da assistência (6), assistência individual (3), assistência integral (3), processo de enfermagem (2), atividade privativa do enfermeiro (1), relação com cuidados indiretos (1), melhorar a assistência (1). Já os núcleos de sentido que emergiram da questão 4 e suas respectivas frequências foram: sistematiza (3), não sistematiza (3), realiza as fases do processo que compõe o formulário da instituição (3), plano de cuidados (3), não realiza prescrição (3), realiza histórico de enfermagem (2), falta conhecimento (2), não é institucionalizado (2), utiliza teoria (1), não utiliza teoria (1), falta educação continuada (1). 
Todos os enfermeiros (100\%) demonstraram ter algum conhecimento sobre a SAE, mas, no entanto, somente metade deles (50\%) afirmou sistematizar a assistência. Os que referiram não sistematizar atribuíram o fato, principalmente, à não institucionalização dessa prática e à falta de conhecimento. Há que se considerar que existe um conflito de conceitos com relação à SAE. "PE, metodologia da assistência e a própria SAE ora apresentam-se como sinônimos e ora apresentam-se com diferentes definições". ${ }^{11: 884}$ Genericamente se referem à forma de organizar a assistência, mas, conceitualmente, não se caracterizam como sinônimos.

Assim como a SAE, a Metodologia da Assistência de Enfermagem (MAE) orienta o profissional na elaboração sistemática da assistência e é o emprego do método científico para solucionar problemas de enfermagem. Sendo assim, é um caminho, um modo lógico de conduzir o trabalho e pode ser considerada um dos elementos da SAE. 5.12
Por sua vez, o PE tem sido confundido com a MAE. 0 PE é um instrumento metodológico e sistemático de prestação de cuidados, que serve à atividade intelectual $\mathrm{e}$ que provê um guia para um determinado estilo de julgamento. ${ }^{13}$

Analisando essas proposições, pode-se considerar que a SAE torna possível a utilização do PE através da operacionalização da MAE. Os enfermeiros que referiram que a SAE não é institucionalizada demonstraram desconhecer que a MAE, embasada em Paim, é a própria sistematização institucionalizada e que deve ser seguida.

Por outro lado, os enfermeiros que referiram sistematizar a assistência (50\%) indicaram em suas respostas ao questionário que realizam todas as três fases da metodologia e se atribuíram um escore alto na escala Likert, com os escores variando de 15 a 11 pontos (Quadro 1).

Quadro 1. Resultados das Escalas de Likert - preenchidas por autopontuação e pelo pesquisador.

\begin{tabular}{|c|c|c|c|c|c|c|c|c|c|c|c|c|}
\hline \multirow{3}{*}{$\begin{array}{l}\text { Ações da gerência do cuidado aos } \\
\text { clientes internados no Serviço de } \\
\text { Infectologia, com base na SAE: } \\
\mathrm{A}=\text { Autopontuação } \\
\mathrm{P}=\text { Pesquisador }\end{array}$} & \multicolumn{12}{|c|}{ SUJEITOS } \\
\hline & \multicolumn{2}{|c|}{ Laranjeira } & \multicolumn{2}{|c|}{ Jabuticabeira } & \multicolumn{2}{|c|}{ Figueira } & \multicolumn{2}{|c|}{ Macieira } & \multicolumn{2}{|c|}{ Mangueira } & \multicolumn{2}{|c|}{ Amendoeira } \\
\hline & $A$ & $P$ & $A$ & $P$ & $\bar{A}$ & $\bar{P}$ & A & $P$ & $\bar{A}$ & $P$ & $A$ & $\bar{P}$ \\
\hline $\begin{array}{l}\text { 1- Realiza o histórico de enfermagem } \\
\text { na ocasião da admissão do cliente na } \\
\text { unidade, a fim de identificar os } \\
\text { problemas de enfermagem? }\end{array}$ & 5 & 1 & 5 & 1 & 2 & 1 & 1 & 1 & 1 & 1 & 1 & 1 \\
\hline $\begin{array}{l}\text { 2- Realiza prescrição de enfermagem } \\
\text { durante a assistência prestada aos } \\
\text { clientes? }\end{array}$ & 3 & 1 & 5 & 1 & 2 & 1 & 4 & 1 & 5 & 1 & 1 & 1 \\
\hline $\begin{array}{l}\text { 3- Realiza evolução de enfermagem, } \\
\text { descrevendo os problemas que foram } \\
\text { solucionados e os que não foram? }\end{array}$ & 4 & 4 & 5 & 3 & 2 & 2 & 4 & 1 & 5 & 4 & 2 & 2 \\
\hline ESCORE TOTAL & 12 & 6 & 15 & 5 & 6 & 4 & 9 & 3 & 11 & 6 & 4 & 4 \\
\hline \multirow[t]{2}{*}{ GRAU DE REALIZAÇÃO } & & & & & & & & & & & & \\
\hline & $\stackrel{\circ}{\stackrel{ }{<}}$ & 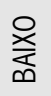 & $\stackrel{ }{\stackrel{一}{<}}$ & 욿 & 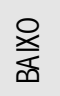 & 앚 & 总 & 잃 & $\stackrel{\circ}{\stackrel{一}{\gtrless}}$ & 음 & 잃 & 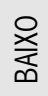 \\
\hline
\end{tabular}

No entanto, a observação sistemática não evidenciou o fato, sendo atribuído pelo pesquisador um escore baixo aos sujeitos, variando de 3 a 6 pontos (Quadro 1). Com relação à realização dos históricos e prescrições de enfermagem, todos os sujeitos receberam escore igual a 1 ponto, ou seja, não realiza. Ao mesmo tempo, não foram encontrados históricos e prescrições de enfermagem nos prontuários analisados na auditoria, explicando a discrepância entre a autopontuação dos sujeitos e a pontuação do pesquisador.

Essa discussão traz à tona um dos grandes problemas evidenciados no estudo: a deficiência dos registros de enfermagem. A falta de registro torna a SAE informal o que atrapalha a realização de todas as etapas. A não realização do histórico compromete a identificação e levantamento dos problemas dos clientes, o que compromete a realização da prescrição e posteriormente da evolução de enfermagem, 0 que torna a MAE incompleta e inoperante, revelando uma contradição entre o que é dito e o que é praticado.

Esse aspecto nos registros comprometeu todas as fases da MAE, pois os sujeitos não realizaram o histórico nem as prescrições de enfermagem, restando então as evoluções de enfermagem para registrar toda a assistência prestada. Os 
registros encontrados nos prontuários se limitavam às evoluções de enfermagem realizadas pelos enfermeiros e por anotações pontuais do estado geral dos clientes realizadas por técnicos e auxiliares de enfermagem da equipe do Serviço.

Com relação às evoluções de enfermagem, quatro sujeitos informaram realizá-las de forma completa. Dois desses informaram que as fazem muitas vezes (Quadro 1). No entanto, $76,92 \%$ dos prontuários continham evoluções de admissão do cliente no serviço incompletas, ou seja, não contemplaram a unidade de origem do cliente, avaliação subjetiva e observações, queixas e relatos, exame físico, sinais vitais, procedimentos realizados, estabelecimento de metas, etc. Em 92,30\% dos prontuários as evoluções diárias também puderam ser consideradas mínimas, com relação aos parâmetros acima descritos.

Por sua vez, em $100 \%$ dos prontuários, a evoluções de alta hospitalar foram incompletas, não informando as condições gerais do cliente, mas apenas o relato breve da sua saída. Para a enfermagem, o descaso com o registro sistemático dos elementos da assistência pode resultar, por um lado, em ausência de visibilidade e de reconhecimento profissional; por outro, o que é talvez mais sério, em ausência ou dificuldade de avaliação de sua prática. ${ }^{14}$

\section{Gerência do cuidado e sua relação com a sistematização da assistência de enfermagem}

Essa categoria refere-se à relação entre a SAE e a gerência do cuidado, de acordo com a opinião dos sujeitos através das respostas dois e três do questionário, comparando com a observação do pesquisador e análise dos prontuários, confrontando com pressupostos teóricos descritos na literatura.

Os núcleos de sentido que emergiram da questão dois e as respectivas frequências com que surgiram foram: cuidados indiretos e diretos (6), organização e supervisão (4), plano de cuidados (4), equipe de enfermagem (2). Já núcleos de sentido que emergiram da questão três e suas respectivas frequências foram: vê relação (6), cuidados diretos e indiretos (6), uma complementa a outra (5), equipe de enfermagem (2), gerência do cuidado incluída na SAE (1), SAE incluída na gerência do cuidado (1).

Quando questionados sobre o que entendiam por gerência do cuidado, $100 \%$ dos sujeitos consideraram que esta inclui cuidados diretos (assistência) e indiretos (ações gerenciais). Ainda, $66 \%$ afirmaram que entre as ações da gerência do cuidado está a organização da assistência através da elaboração de um plano de cuidados. A noção fragmentada de que o trabalho de enfermagem envolve o espaço dos cuidados assistenciais e o da administração da assistência é hoje ultrapassada pela noção de integralidade e relações múltiplas interativas do cuidar, do gerenciar e do educar.

Sabe-se que a enfermagem, ao longo de sua história, tem enfrentado inúmeros desafios na construção de sua identidade, na apropriação do seu objeto de trabalho nuclear - o cuidado de enfermagem - e na obtenção do reconhecimento e valorização do seu fazer. Ela tem se empenhado junto às instituições de saúde para desenvolver as atividades administrativas inerentes à gerência não mais como um trabalho subdividido, mas sim como trabalho articulado com os demais serviços, em uma relação de troca e ajuda, que envolvem diversos atores. ${ }^{15}$

Há, no entanto, uma desarticulação entre a assistência e a gerência, pois é possível reconhecer que os processos de cuidar e de administrar quase não se tocam, configurando eixos distintos que têm corrido em paralelo. ${ }^{16}$ No Serviço de Infectologia essa distância é evidenciada, sobretudo, através da desarticulação das fases do PE. A realização e coordenação dessas atividades seriam de fato $o$ link entre a assistência e a gerência do cuidado.

Não será possível efetivar mudanças significativas no sistema de saúde caso não haja participação efetiva de todos os profissionais para a reorganização dos serviços. É essencial que o enfermeiro se aproprie dos instrumentos gerenciais para transformar o processo de cuidar. ${ }^{17}$ Deduz-se que os enfermeiros, ao considerarem que gerenciar a assistência é, entre outras coisas, organizar o cuidado, estão construindo uma relação entre a SAE (consideradas também como uma forma de organizar o cuidado) e a própria gerência do cuidado. Assim, a SAE é um instrumento que auxilia o gerente a nortear e avaliar a assistência que sua equipe prestou, sendo considerada uma ferramenta gerencial. ${ }^{2}$

Compreende-se a SAE como instrumento metodológico tanto para favorecer o cuidado quanto para organizar as condições para que este ocorra. Ela é simplesmente a forma como o enfermeiro vem buscando agregar cientificidade para organizar as ações de prestação do cuidado. A utilização da SAE possibilita ao enfermeiro realizar com maior eficácia ações de supervisão, de avaliação e de gerenciamento dos cuidados prestados, bem como acompanhar os resultados das ações implementadas.

Partindo desta premissa é que se localiza a SAE como um dos instrumentos do processo de trabalho assistencial do enfermeiro que pode contribuir para assegurar a qualidade do cuidado, desde que não seja entendido como a totalidade da assistência de enfermagem, pois esta contempla uma ampla gama de instrumentos que inclui a comunicação, a interação e a articulação das dimensões assistencial e gerencial, dentre outros. Sendo assim, entende-se também que a SAE não pode ser concebida como a totalidade do gerenciamento, pois isso se configura um discurso ideológico e reducionista, que restringe o processo gerencial somente a esse instrumento, o que não é verdade.

A gerência do cuidado é a ferramenta que o enfermeiro utiliza para coordenar e sistematizar a prestação do cuidado direto, devendo ser planejado, analisado e avaliado não perdendo de vista que o sucesso da gerência só se é alcançado através das pessoas e em uma interação humana constante. 
Neste contexto, cabe ao enfermeiro implementar a SAE, prever e prover o serviço de recursos materiais e capital humano qualificados para que o cuidado se efetive.

\section{CONCLUSÃO}

No percurso da análise foi possível identificar, pela resposta dos sujeitos, que eles compreendem o que éSAE e a gerência do cuidado e que enxergam uma relação entre as duas. No entanto, somente $50 \%$ dos enfermeiros referiram sistematizar utilizando a MAE de Paim. Os demais enfermeiros informaram que não sistematizam por não ser institucionalizado, não sendo preparados para isso. Esses desconhecem que a utilização deste metodologia corresponde à própria SAE institucionalizada.

De outra forma, a observação dos sujeitos e a análise documental demonstrou uma lacuna entre o que é dito e o que é realizado na prática. Em $100 \%$ dos prontuários constatou-se que a MAE foi aplicada de forma incompleta, pois não contemplou o levantamento de problemas no histórico e as prescrições de enfermagem. As evoluções, por sua vez, se deram de forma incompleta na maioria das vezes. É importante esclarecer aos enfermeiros que, utilizando os impressos de enfermagem disponiveis na instituição, já estariam de fato contribuindo para SAE implementada. Ou seja, a SAE foi institucionalizada, mas talvez ainda falte treinamento, apoio e/ou incentivo para que a mesma se efetive entre os profissionais.

Apesar de os sujeitos considerarem que a SAE tem relação com a gerência do cuidado, não se pode afirmar que ela seja uma ferramenta gerencial em uso. Embora existam dificuldades na implementação da SAE como um instrumento para o processo de trabalho, acredita-se que, ao utilizá-lo nas atividades assistenciais e articulá-lo com as atividades gerenciais, a prática profissional do enfermeiro ficará mais bem caracterizada sem a cisão entre o cuidar e o administrar.

Esse estudo mostrou que os enfermeiros do Serviço de Infectologia, sendo todos responsáveis pela gerência administrativa e burocrática da unidade e agirem organizando a assistência prestada, provendo meios e recursos para que 0 cuidado seja possível e capacitando sua equipe para atuar seguindo um modelo de assistência humano, integral e com qualidade, entre outras coisas, podem ser considerados gerentes do cuidado. No entanto, a SAE não faz parte das suas ferramentas gerenciais. Dessa forma, não se pode considerar que a $S A E$, enquanto uma ferramenta da gerência do cuidado, serve de base para as ações de cuidar.

Há também que se considerar que "não há estudo publicado na literatura nacional que demonstre que a implementação da SAE, no contexto dos hospitais brasileiros, garanta a qualidade da assistência de enfermagem" e que "existem poucos artigos que relacionem a SAE com as atividades gerenciais desenvolvidas pelo enfermeiro". 11:884 Ou seja, a própria gerência do cuidado ainda não se atentou e publicou conhecimentos sobre o fato de que a SAE pode ser vista como uma ferramenta gerencial que agrega valor ao trabalho do enfermeiro ou que pelo menos organiza sua execução e facilita sua avaliação.

Não se objetivou apontar falhas na atuação dos profissionais. Reconhece-se que falta capacitação, educação permanente e cobrança por parte das chefias superiores e da própria instituição para a realização de uma assistência realmente sistematizada. De agora em diante, o que precisa ser trabalhado é a consciência dos enfermeiros para que utilizem a SAE, enquanto uma ferramenta gerencial, para embasar a assistência que prestam e assim poderem avaliar resultados.

Para começar, recomendam-se algumas estratégias que possibilitariam um feedback às gerências dos serviços de enfermagem, a saber: organizar grupos de estudos sobre a SAE, articulando seus saberes com a gerência do cuidado; realizar oficinas com as equipes para elaborar um projeto de modificação do impresso da SAE utilizado, caso necessário; construir e avaliar em conjunto o impresso, com participação de todos, visando à elaboração de um instrumento que funcione e atenda às necessidades da equipe; instituir esse impresso e fazer o acompanhamento da implementação, sem perder de vista que esse processo leva tempo; e, por fim, elaborar um projeto de treinamento e capacitação em metodologia da assistência e execução da SAE.

Faz-se também necessário que os enfermeiros revisem periodicamente as anotaç̃̃es feitas, com o propósito de orientar, esclarecer e reforçar o conhecimento dos técnicos e auxiliares, especialmente sobre aspectos que são relevantes para favorecer a qualidade da assistência visando concretizar o crescimento de toda a equipe.

Os fatores que dificultam a utilização da SAE devem-se à falta de preparo dos enfermeiros sobre o método. Portanto, para que a atividade se modifique e eles compreendam a SAE não como receita pronta, mas sim como algo que pode auxiliar e direcionar o trabalho em benefício do cuidado, é importante um acordo e a compreensão da equipe e também contar com um programa de educação permanente bem estruturado, que considere os fatores externos da instituição, bem como contemplar a possível resistência do pessoal incrédulo, que nega a validade que $\mathrm{S} S \mathrm{E}$. oferece à profissão, como metodologia científica, que vem reforçar a autonomia profissional do enfermeiro. ${ }^{18}$

\section{REFERÊNCIAS}

1.Conselho Federal de Enfermagem- CFB. [site de internet]. Decreto $\mathrm{n}^{\circ}$ 94.406, de 08 de junho de 1987. Regulamenta a Lei $n^{\circ} 7.498$, de 25 de junho de 1986 sobre o exercício profissional da enfermagem. [citado 2009 jul 02]. Disponível em: http://www.portalcofen.gov.br/2007/ materias.asp?ArticlelD=26\&section $\mid \mathrm{D}=32$. 
2.Christovam BP. Gerência do cuidado de enfermagem em cenários hospitalares: a construção de um conceito [tese]. Rio de Janeiro: Escola de Enfermagem Anna Nery, Universidade Federal do Rio de Janeiro; 2009.

3.Gonçalves LRR, Nery IS, Nogueira LT, Bonfim EG. 0 desafio de implantar a sistematização da assistência de enfermagem sob a ótica do discente. Esc Anna Nery 2007; [citado 2011 abr 21] ; 11(3): 459-65. Disponível em: http://www.scielo.br/scielo.php?script=sci_arttext\&pid=S141481452007000300010 \&lng=en. doi: 10.1590/S141481452007000300010 .

4.Conselho Federal de Enfermagem-CFB. [site de Internet]. Resolução COFEN n 358/2009. Dispõe sobre a sistematização da assistência de enfermagem e a implementação do processo de enfermagem em ambientes, públicos ou privados, em que ocorre o cuidado profissional de enfermagem, e dá outras providências. [citado 2010 fev 08]. Disponível em: http://www.portalcofen.gov.br/Site/2007/ materias.asp?ArticlelD $=10113$ \&section $\mid \mathrm{D}=34$

5.Silvino ZR. 0 desgaste mental no trabalho dos enfermeiros: entre o real e o prescrito [tese] Rio de Janeiro: Escola de Enfermagem Anna Nery, Universidade Federal do Rio de Janeiro; 2002.

6.Bomfim MES. Assistência de enfermagem: uma contribuição à aplicação do método Lygia Paim. Niterói: Ferraz; 2000.

7.Yin RK. Estudo de caso: planejamento e métodos. $3^{\mathrm{a}}$ ed. Porto Alegre: Bookman; 2005.

8.Polit DF, Beck CT, Hungler BP. Fundamentos de pesquisa em enfermagem. $5^{\mathrm{a}}$ ed. Porto Alegre: Artes Médicas; 2004.

9.Richardson RJ. Pesquisa social: métodos e técnicas. São Paulo: Atlas; 1999.

10.Bardin L. Análise de conteúdo. 3ª ed. Lisboa: Ed 70; 2008.

11.Fuly PSC, Leite JL, Lima SBS. Correntes de pensamento nacionais sobre sistematização da assistência de enfermagem. Rev Bras Enferm. 2008 nov/dez; 61(6): 883-87.

12.Leopardi MT. Teoria e método em assistência de enfermagem. $2^{\mathrm{a}}$ ed. Florianópolis: Soldasof; 2006.

13.Cruz JG. A gerência de enfermagem como instrumento de transformação da assistência em rede básica de saúde [trabalho de conclusão de curso]. Niterói: Escola de Enfermagem Aurora de Afonso Costa, Universidade Federal Fluminense; 2004

14.Garcia TR, Nóbrega MML. Processo de enfermagem e os sistemas de classificação dos elementos da prática profissional: instrumentos metodológicos e tecnológicos do cuidar. In: Santos I, Figueiredo NMA, Padilha MICS, Souza SROS, Machado WCA, Cupello AJ. Enfermagem assistencial no ambiente hospitalar: realidade, questões e soluções. São Paulo: Atheneu; 2004.

15.Prochnow AG, Leite IL, Erdmann AL. Teoria interpretativa de Geertz e a gerência do cuidado: visualizando a prática social do enfermeiro. Rev Latino-am Enfermagem. 2005; 13(4): 583-90.
16.Ferraz CA. As dimensões do cuidado em enfermagem: enfoque organizacional. Acta Paul Enferm. 2000; 13(n esp 1): 91-7.

17.Campos GWS. Considerações sobre o processo de administração e a gerência dos serviços de saúde. In: Campos GWS, Merhy EE, Nunes ED. Planejamento sem normas. São Paulo: Hucitec; 1994.

18. Hermida PMV. Desvelando a implementação da sistematização da assistência de enfermagem. Rev Bras Enferm. 2004 nov/dez; 57 (6): 733-37. 